10. Haramaki N, Ikeda H, Takajo $Y$, et al. Long-term smoking causes nitroglycerin resistance in platelets by depletion of intraplatelet glutathione. Arterioscler Thromb Vasc Biol 2001; 21: 1852-6.

11. Markovitz JH, Tolbert L, Winders SE. Increased serotonin receptor density and platelet GPIIb/IIIa acti- vation among smokers. Arterioscler Thromb Vasc Biol 1999; 19: 762-6.

12. Li Y, Woo V, Bose R. Platelet hyperactivity and abnormal calcium homeostasis in diabetes mellitus. Am J Physiol Heart Circ Physiol 2001; 280: 1480-9.
13. Pietraszek MH, Takada Y, Takada A, et al. Blood serotonergic mechanisms in type 2 (non-insulin-dependent) diabetes mellitus. Thromb Res 1992; 66: 765-74.

\title{
Inverse correlation between phenylacetate hydrolase activity of the serum PONI pro- tein and homocysteinemia in humans
}

\section{Dear Sir,}

Hyperhomocysteinemia is an independent risk factor for cardiovascular disease and stroke, while also being a risk factor for neurodegenerative and renal disorders. However, the actual pathological mechanisms of increased serum levels of Homocysteine (Hcy) or its metabolites in human remain elusive.

The determinants of plasma Hcy concentration are both genetic as well as environmental. Moderate Hcy elevations can be due to homozygosity of the $677 \mathrm{~T}$ allele of the MTHFR gene or nutritional deficiencies in the cofactors (vitamin B6 or B12) or substrate (folate) of the enzymes involved in Hcy metabolism. Severe hyperhomocysteinemia is often caused by inborn error of metabolism such as in Cystathionine Beta Synthase (CBS) deficiency.

Hcy is synthesized during the conversion of dietary methionine to cysteine. Once synthesized, Hcy can be recycled to me-

\section{Correspondence to:}

Jean François Chassé

INSERM UMR S-490

Université Paris V

45 rue des saints Pères

Paris, Cedex 6

75270 France

Tel.: 33 | 42862073, Fax: 33 | 42862072

E-mail: jean-francois.chasse@univ-paris5.fr

Received July 30, 2004

Accepted after revision September 9, 2004

Thromb Haemost 2005; 93: I82-3 thionine by the remethylation pathway or converted into cystathionine by the transulfuration pathway. Hcy is also metabolically converted to form homocysteine thiolactone (HTL) by a metabolic error-editing process in which Hcy is inactivated by methionyl-tRNA synthethase and is released as free HTL. HTL has been implicated in the pathology of hyperhomocysteinemia probably through increased protein homocysteinylation (1).

Paraoxonase 1 (PON1) is secreted by the liver and is a protein component of the high-density lipoproteins. Paraoxonase displays esterase activity and can hydrolyse various exogenous and endogenous substrates (2). It degrades oxidized lipids and is thus considered as a protective anti-oxidant enzyme. Several studies have shown increased susceptibility to cardiovascular disease in individuals carrying polymorphisms in the PON1 gene (3). Interestingly, paraoxonase has been recently shown to degrade HTL (4) and thus could contribute to the detoxification of this metabolite of Hcy. Moreover, in the murine model of severe hyperhomocysteinemia due to CBS deficiency, the activity of PON1 in the liver was downregulated 3-fold (5). The moderate to severe hyperhomocysteinemia in mice, caused by a hyperhomocysteinemic diet or by a genetic deficiency in CBS, is associated with reduced liver PON1 activity, with a downregulation of PON1 mRNA in the liver (6). We therefore hypothesized that homocysteine serum levels could influence serum paraoxonase activity in humans.

Blood samples from 112 subjects with various homocysteinemia were recruited from the Hospital Europeen Georges Pompidou (HEGP-France) in the different clinical units of hos-
Figure I: Relation between homocysteine concentration and relative phenylacetate hydrolase activity.

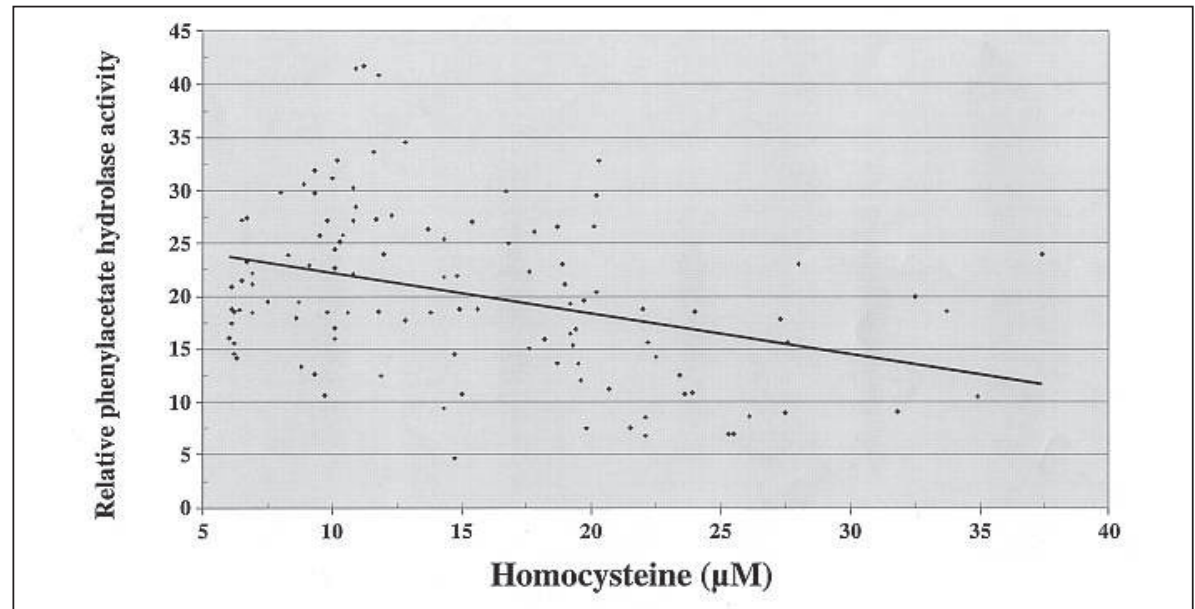


pitalisation. Phenylacetate hydrolase activity was assayed on these blood samples using serum vacutainer tubes to avoid calcium chelation. The colorimetric assay was run as described (5) using phenylacetate as substrate. Phenylacetate degrading activity of PON1 is independent of the coding region polymorphisms (position 55 and 192) of this gene and thus is more reflective of the concentration of PON1 protein present (7). It is unclear whether this activity reflects the actual activity of the PON1 towards endogenous substrates such as HTL. Neither HTL degrading activity, nor HTL concentrations have been assayed in this study. The precise HTL level in human plasma has been reported for six persons (8).

An inverse correlation was found between Hcy concentration and phenylacetate hydrolase activity $(\mathrm{r}=0.36, \mathrm{y}=-0.34 \mathrm{x}+$ $22,01, \mathrm{ddl}=110, \mathrm{t}=4,09, \mathrm{p}<0.001-$ Fig. 1). Thus, the level of Hcy appears to be one of the determinants of phenyl-acetate hydrolase activity in serum. While several mechanisms could account for such an effect, the observations made in hyperhomocysteinemic mice suggest that Hcy could negatively regulate PON1 gene expression in the liver which would result in decreased serum PON1 activity. It is unknown whether this Hcy effect is direct or not, however, the fact that Hcy is itself a product of PON1 activity suggests an autoregulatory mechanism whereby increased Hcy concentrations lead to the retroinhibition of the enzyme gene expression.
The observations shown here could have implications for the delineation of the mechanism of Hcy action. Indeed, it is known that altered PON1 activity as observed in individuals carrying the polymorphisms at position 55 and 192 constitutes a susceptibility marker for cardiovascular disease. This is likely due to the lipoproteins-protective anti oxidant effect of PON1 and possibly to its ability to degrade HTL. The repression of serum PON1 activity could be one of the steps leading to Hcy toxicity. Alternatively, our data also suggest that PON1 level could be an additional determinant of the Hcy plasma concentration.

\section{Acknowledgments}

This work was supported by the European Union Grant (QLRT-2001-00816) and a grant "vin et santé". I thank Anna Kostrzack for their technical assistance

\section{Nathalie Janel ${ }^{2}$, Karine Robert ${ }^{2}$, Karine Demuth ${ }^{3}$, Cédric Gouédard', Robert Barouki', 3, Jean François Chassél, 3 IINSERM UMR S-490, Université Paris V, Paris, France \\ ${ }^{2}$ EA 3508, Université Paris 7, Paris, France}

${ }^{3}$ Service de Biochimie, Hopital Europeen Georges Pompidou, Paris, France

\section{References}

1. Glowacki R, Jakubowski H Cross-talk between CYS-34 and lysine residues in human serum albumin revealed by N-homocysteinylation. J Biol Chem 2004; 279: 10864-71.

2. Jakubowski H, Ambrosius WT, Pratt JH. Genetic determinants of homocysteine thiolactonase activity in humans: implications for atherosclerosis. FEBS Lett 2001; 491: 35-9.

3. Voetsch B, Loscalzo. Genetic determinants of the arterial thrombosis. Arterioscler Thromb Vasc Biol 2004; 24: 216-29.
4. Jakubowski H. Calcium-dependant human serum homocysteine thiolactone hydrolase. J Biol Chem 2000; 275: 3957-62.

5. Robert K, Chassé JF, Santiard-Baron D et al. Altered gene expression in liver from a murine model of hyperhomocysteinemia. J Biol Chem 2003; 278 . 31504-11.

6. Janel N, Robert K, Chabert $\mathrm{C}$ at al. Mouse liver paraoxonase-1 gene expression is downregulated in hyperhomocysteinemia. Thromb Haemost 2004; 92 221-2.
7. Jarvik GP, Hatsukami TS, Carlson C et al. Paraoxonase activity, but not haplotype utilizing the linkage disequilibrium structure, predicts vascular disease. Arterioscler Thromb Vasc Biol 2003; 23: 1465-71.

8. Jakubowski H. The determination of homocysteine-thiolactone in biological samples. Anal Biochem 2002; 308: 112-9.

\section{Elastic stockings, hydroxyethylrutosides or both for the treatment of post-thrombotic syndrome}

\section{Dear Sir,}

At least one of every three patients with deep-vein thrombosis (DVT) of the lower extremities will develop post-thrombotic sequelae within 5 years $(1,2)$. The established post-thrombotic

\footnotetext{
Correspondence to:

Paolo Prandoni

Department of Medical and Surgical Sciences

$2^{\text {nd }}$ Chair of Internal Medicine

University of Padua, Italy

Tel.: +39-049-8212656, Fax: +39-049-821266|

E-mail: paoloprandoni@tin.it

Received August 15, 2004

Accepted after October 19, 2004
}

Thromb Haemost 2005; 93: I 83-5 syndrome (PTS) remains a significant cause of chronic ill health, with considerable socio-economic consequences for both the patient and the health care services (3).

In contrast with the extensive documentation available on epidemiology, diagnosis, and prevention of PTS, little is known about the treatment of this common complication of DVT (4). Once the PTS is established, treatment options are aimed at limiting progression to an ulcer with the use of graduated elastic compression stockings (ECS), which reduce venous hypertension and improve microcirculation (5). Besides compression therapy, rutosides are commonly used for prevention and treatment of several manifestations of chronic venous insufficiency (6). In a prospective, controlled, randomized pilot study, we compared 